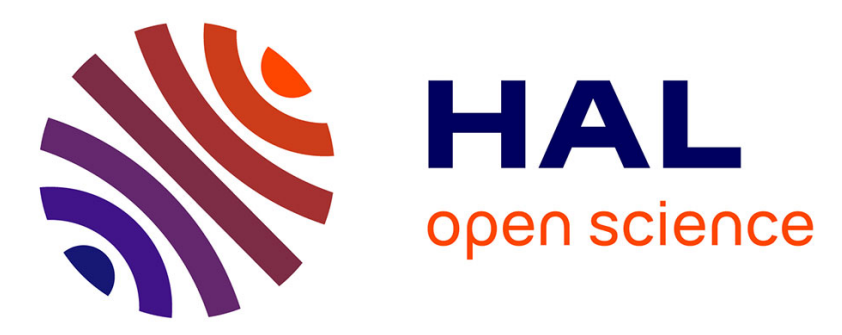

\title{
Generalized formulation of the interactions between soft spheres
}

Fernando Alonso-Marroquín, Sean Mcnamara

\section{To cite this version:}

Fernando Alonso-Marroquín, Sean Mcnamara. Generalized formulation of the interactions between soft spheres. The European Physical Journal. Special Topics, 2014, 223 (11), pp.2227. 10.1140/epjst/e2014-02260-5 . hal-01140641

\section{HAL Id: hal-01140641 \\ https://hal.science/hal-01140641}

Submitted on 9 Apr 2015

HAL is a multi-disciplinary open access archive for the deposit and dissemination of scientific research documents, whether they are published or not. The documents may come from teaching and research institutions in France or abroad, or from public or private research centers.
L'archive ouverte pluridisciplinaire HAL, est destinée au dépôt et à la diffusion de documents scientifiques de niveau recherche, publiés ou non, émanant des établissements d'enseignement et de recherche français ou étrangers, des laboratoires publics ou privés. 


\title{
Generalized formulation of the interactions between soft spheres
}

\author{
Fernando Alonso-Marroquín · Sean McNamara
}

Received: June 25, 2014

\begin{abstract}
The goal of this paper is to identify the most general formulation that consistently links the different degrees of freedom in a contact between spherical soft particles. These contact laws have two parts: a set of "generalized contact velocities" that characterize the relative motion of the two particles, and a set of "generalized contact forces" that characterize the interparticle forces.

One well known constraint on contact models is that the contact velocities must be objective. This requirement fixes the number of linearly independent contact velocities. We also present a previously unnoticed (in this context) constraint, namely, that the velocities and forces must be related in such a way that the stiffness matrix is symmetric. This constraint also places restrictions on the coupling between the contact forces.

Within our generalized contact model, we discuss the expression for rolling velocity that need to be used in the calculation of rolling resistance, and the risk or producing perpetual mobile when other expressions of rolling velocity are using instead.
\end{abstract}

Keywords Rolling resistance · discrete element modeling $\cdot$ soft sphere

\section{Introduction}

Soft spheres discrete element modeling (DEM) has been used by physicists, engineers, geo-technical/-physical and many other researchers worldwide for almost forty years $[1 ; 2 ; 3]$ to model particle systems. Simulations of

S. McNamara

Institut de Physique de Rennes, Université de Rennes 1, France

F. Alonso-Marroquín, School of Civil Engineering, The University of Sydney, Australia soft spheres involve interactions via visco-elastic forces $[2 ; 4 ; 5 ; 6]$, rolling resistance that accounts the deformation of the particles at the contact $[7 ; 8 ; 9]$, or mimics the effect of particle shape $[10 ; 11]$, and torsion moments to model inter-particle bonds $[12 ; 13 ; 14]$. Yet the question of how to correctly and best model the interactions is still open to discussion.

Most contact models are based on the strong assumption of decoupling of the constitutive models across the degrees of freedom: normal, tangential, sliding, rolling (bending) and torsion. (torsion refers to the twisting at the contact, and rolling corresponds to bending in cohesive contacts)

Even with this simplification, the best way to define a rolling velocity and rolling resistance - how much two spheres roll relative to each other, and what is the resistance/reaction to this motion - has not been established so far. Numerous papers $[15 ; 16 ; 17 ; 18 ; 19$; $20 ; 13 ; 21 ; 22]$, have proposed different models for the rolling velocity,

but it is not clear how one should choose among the different alternatives. Even after requiring that the rolling velocity be objective, that is, independent of the reference frame in which the motion of the touching spheres is measured $[13 ; 18 ; 19 ; 20]$, many alternatives remain. For example, one thorough study [19] considered four different objective rolling velocities, and found that they are all roughly equivalent. Nevertheless, the literature seems to converge towards formulations with simplified version of rolling resistance $[13 ; 23 ; 24 ; 25]$.

In this paper, we show that the symmetry of the stiffness matrix [26] imposes a relation between the rolling velocity and the contact moment (or rolling resistance), i.e. the combination of contact forces and moments that the touching grains exert on one another in response to the rolling motion. Specifically, we show below that if 
rolling generates equal and opposite contact moments, then the rolling and torsion velocity is uniquely determined - and it must be of the form proposed by Jiang [24] and Luding [13]. More generally, we show that the degrees of freedom can be coupled, but this must be done in a symmetric way.

In Sec. 2 we present the general form a contact law must have by first setting out our notation in Sec. 2.1, discussing objectivity in Sec. 2.2, and the conservation of linear and angular momentum in Sec. 2.3. These constraints on contact laws have already been discussed. Then we introduce the new constraint on the contact laws in Sec. 2.4 and 2.5.

In Sec. 3 we present perhaps the simplest model that obeys all the conditions described above. Finally, in Sec. 4, we discuss the consequences of our work on other models.

\section{General considerations}

In this section, we present the constraints that all contact laws must follow.

\subsection{What is a contact law?}

Consider the kinematics of two spheres in contact (see Figure 1) as a generalized vector in a twelve dimensional velocity space.

$\mathbf{V}=\left[\begin{array}{l}\mathbf{v}_{1} \\ \mathbf{v}_{2} \\ \boldsymbol{\omega}_{1} \\ \boldsymbol{\omega}_{2}\end{array}\right]$

with $\mathbf{v}_{i}$ being the translational velocities of particles $i=1,2$, and $\boldsymbol{\omega}_{i}$ their rotational, angular velocities. Note that we adopt here the freedom to keep entries of the vectors/matrices with different units, which is a wellestablish practice in structural mechanics [27]. Having vectors with different units does not pose a problem as long as we do not calculate quantities with inconsistent units. For example, we cannot calculate the length of $\mathbf{V}$ using the traditional dot product.

The interaction (force and torque) between the two particles is given by a generalized force-vector

$\mathbf{F}=\left[\begin{array}{c}\mathbf{F}_{12} \\ \mathbf{F}_{21} \\ \mathbf{T}_{12} \\ \mathbf{T}_{21}\end{array}\right]$

where $\mathbf{F}_{i j}$ is the force on particle $i$ due to particle $j$ and $\mathbf{T}_{i j}$ is the torque on particle $i$ due to particle $j$.

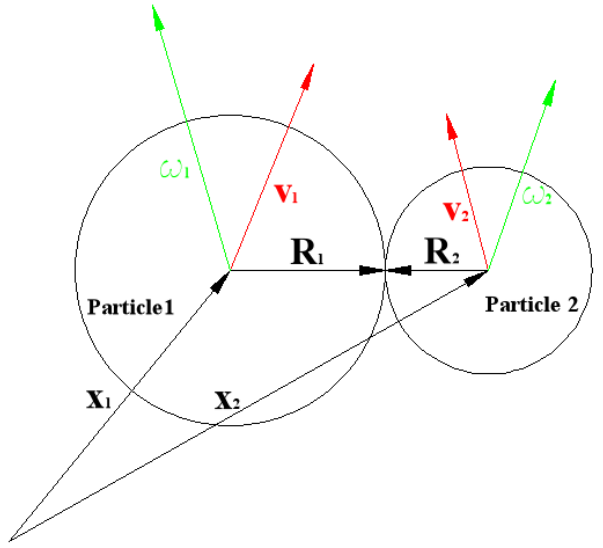

Fig. 1 Velocities of particle 1 and particle 2 in contact

A contact model simply gives a relation between $\mathbf{F}$ and $\mathbf{V}$. One begins by calculating certain linear combinations of the components of $\mathbf{V}$ that represent the relative motion of the spheres:

$\mathbf{v}=\mathbf{C V}$

where $\mathbf{C}$ is a transformation matrix. The different components of $\mathbf{v}$ contain the different types of relative motion: normal and tangential velocity, sliding and rolling, for example. Other combinations are in theory possible.

Next the contact forces must be calculated with the contact model, that we will represent by a vector $\mathbf{f}$. These forces are calculated from the current and past values of $\mathbf{v}$. The integral of $\mathbf{v}$, representing some kind of "contact springs", are almost always used:

$\boldsymbol{\delta}=\int_{t_{0}}^{t} \mathbf{v}\left(t^{\prime}\right) d t^{\prime}$

where $t_{0}$ is the time where the contact forms. The components of $\boldsymbol{\delta}$ give the accumulated amount of each kind of contact displacement. For example, if one of the components of $\mathbf{v}$ is the normal relative velocity, then the corresponding component of $\boldsymbol{\delta}$ is the overlap.

In the linear spring model, a contact force depends linearly on each component of $\boldsymbol{\delta}$ :

$\mathbf{f}=\mathbf{k} \delta$.

where $\mathbf{k}$ is a diagonal matrix whose non-zero entries are spring stiffnesses.

In other models (the Hertz law for example), $\mathbf{f}$ is a nonlinear function of $\boldsymbol{\delta}$. Even in these cases, however, we are usually able to linearize the contact forces about the current value of $\boldsymbol{\delta}$, i.e.,

$\mathbf{f}\left(t_{0}+\Delta t\right)=\mathbf{f}\left(t_{0}\right)+\mathbf{k} \int_{t_{0}}^{t_{0}+\Delta t} \mathbf{v}(t) d t$ 
so that the linear spring model has a wider significance than its simple nature might suggest.

Finally, the contact forces must be applied to the particles. Since $\mathbf{f}$ represents interaction forces or torques applied to the grains, the relation between $\mathbf{f}$ and $\mathbf{F}$ is linear:

$$
\mathbf{F}=\mathbf{C}^{*} \mathbf{f}
$$

where $\mathbf{C}^{*}$ is another matrix.

In conclusion, therefore, a contact model must specify:

1. A matrix $\mathbf{C}$ which converts the particle velocities $\mathbf{V}$ to the contact velocities $\mathbf{v}$,

2. An algorithm for calculating the contact forces $\mathbf{f}$ from the contact velocities v. For small displacements, this relation can often be linearized, so that $\Delta \mathbf{f}=\mathbf{k} \int \mathbf{v} d t^{\prime}$

3. A matrix $\mathbf{C}^{*}$ that relates the contact forces $\mathbf{f}$ to the total interaction forces $\mathbf{F}$.

\subsection{Objectivity}

The dimension of $\mathbf{v}$ is fixed by the requirement that the contact velocities be objective, that is, invariant under both Galilean and Jaumann transformations. Under a Galilean transformation, a constant is added to the linear velocities:

$\mathbf{V} \rightarrow \mathbf{V}^{\prime}=\mathbf{V}+\mathbf{U}_{G}=\left[\begin{array}{c}\mathbf{v}_{1} \\ \mathbf{v}_{2} \\ \boldsymbol{\omega}_{1} \\ \boldsymbol{\omega}_{2}\end{array}\right]+\left[\begin{array}{c}\mathbf{u}_{G} \\ \mathbf{u}_{G} \\ 0 \\ 0\end{array}\right]$

where $\mathbf{u}_{G}$ is any vector in $\mathbb{R}^{3}$. Thus all possible vectors $\mathbf{U}_{G}$ form a 3-dimensional subset in $\mathbb{R}^{12}$.

Requiring $\mathbf{v}$ to be objective leads to

$\mathbf{v}=\mathbf{C V}=\mathbf{C}\left(\mathbf{V}+\mathbf{U}_{G}\right) \Rightarrow \mathbf{C U}_{G}=0$.

Thus $\mathbf{C}$ is rank 9, at most.

Under a Jaumann transformation, a constant is added to both linear and angular velocities:

$\mathbf{V} \rightarrow \mathbf{V}^{\prime}=\mathbf{V}+\mathbf{U}_{J}=\left[\begin{array}{l}\mathbf{v}_{1} \\ \mathbf{v}_{2} \\ \boldsymbol{\omega}_{1} \\ \boldsymbol{\omega}_{2}\end{array}\right]+\left[\begin{array}{c}\boldsymbol{\omega}_{J} \times\left(\mathbf{x}_{1}-\mathbf{x}_{0}\right) \\ \boldsymbol{\omega}_{J} \times\left(\mathbf{x}_{2}-\mathbf{x}_{0}\right) \\ \boldsymbol{\omega}_{J} \\ \boldsymbol{\omega}_{J}\end{array}\right]$

where $\mathbf{x}_{0}$ and $\boldsymbol{\omega}_{J}$ are vectors in $\mathbb{R}^{3}$. Thus all possible vectors $\mathbf{U}_{J}$ form a 3 -dimensional subset in $\mathbb{R}^{12}$.

Requiring $\mathbf{v}$ to be objective leads to

$\mathbf{v}=\mathbf{C V}=\mathbf{C}\left(\mathbf{V}+\mathbf{U}_{J}\right) \Rightarrow \mathbf{C} \mathbf{U}_{J}=0$.
Thus, solid body rotations of the two grains form a second three dimensional family of transformations that must also be in the null space of $\mathbf{C}$. This lowers the rank of $\mathbf{C}$ to 6 . Imposing objectivity means that we will have at most six linearly independent contact velocities. We can therefore take $\mathbf{v} \in \mathbb{R}^{6}$ without loss of generality.

\subsection{Conservation of momentum}

The conservation of momentum imposes a similar constraint on $\mathbf{f}$. If the two particles do not interact with their environment, their total momentum is constant:

$m_{1} \mathbf{v}_{1}+m_{2} \mathbf{v}_{2}=$ Const

Differentiating this equation with respect to time gives

$m_{1} \frac{d \mathbf{v}_{1}}{d t}+m_{2} \frac{d \mathbf{v}_{2}}{d t}=0$

and combining with Newton's second law

$m_{1} \frac{d \mathbf{v}_{1}}{d t}=\mathbf{F}_{12} \quad m_{2} \frac{d \mathbf{v}_{2}}{d t}=\mathbf{F}_{21}$

gives Newton's third law:

$\mathbf{F}_{12}+\mathbf{F}_{21}=0$.

The vectors in this equation have three components, and thus three constraints are applied to the vector $\mathbf{F}$. This means that $\mathbf{F}$ belongs to an at most ninedimensional subspace of $\mathbb{R}^{12}$.

Now let us consider the conservation of angular momentum about an arbitrary point $\mathbf{x}_{0}$ :

$$
\begin{aligned}
m_{1}\left(\mathbf{x}_{1}-\mathbf{x}_{0}\right) \times \mathbf{v}_{1}+m_{2} & \left(\mathbf{x}_{2}-\mathbf{x}_{0}\right) \times \mathbf{v}_{2} \\
& +I_{1} \boldsymbol{\omega}_{1}+I_{2} \boldsymbol{\omega}_{2}=\text { Const. }
\end{aligned}
$$

Differentiating by time we have

$$
\begin{aligned}
& m_{1}\left(\mathbf{x}_{1}-\mathbf{x}_{0}\right) \times \frac{d \mathbf{v}_{1}}{d t}+ m_{2}\left(\mathbf{x}_{2}-\mathbf{x}_{0}\right) \times \frac{d \mathbf{v}_{2}}{d t} \\
&+I_{1} \frac{d \boldsymbol{\omega}_{1}}{d t}+I_{2} \frac{d \boldsymbol{\omega}_{2}}{d t}=0 .
\end{aligned}
$$

Note that the temporal derivative of $\mathbf{x}_{1}$ dissappears because $d \mathbf{x}_{1} / d t \times \mathbf{v}_{1}=\mathbf{v}_{1} \times \mathbf{v}_{1}=0$.

Using Newton's second and third laws, Eq. (14) and (15), together with

$I_{1} \frac{d \boldsymbol{\omega}_{1}}{d t}=\mathbf{T}_{12} \quad I_{2} \frac{d \boldsymbol{\omega}_{2}}{d t}=\mathbf{T}_{21}$,

we have

$\left(\mathbf{x}_{1}-\mathbf{x}_{2}\right) \times \mathbf{F}_{12}+\mathbf{T}_{12}+\mathbf{T}_{21}=0$.

This is a second equation that imposes three conditions on the components of $\mathbf{F}$. Thus all physically admissible values of $\mathbf{F}$ lie in a six-dimensional subspace of $\mathbb{R}^{12}$. Without loss of generality, we can therefore take $\mathbf{f} \in \mathbb{R}^{6}$. 


\subsection{Power}

We now turn our attention to the relation between the forces and the velocities. These two quantities appear together in the expression for the rate of work done by the contact forces.

Expressions for the kinetic energy can be obtained by taking the scalar product of the equations of motion Eqs. (14) and (18) with the corresponding velocity:

$m_{i} \frac{d \mathbf{v}_{i}}{d t} \cdot \mathbf{v}_{i}=\mathbf{F}_{i j} \cdot \mathbf{v}_{i}, \quad I_{i} \frac{d \boldsymbol{\omega}_{i}}{d t} \cdot \boldsymbol{\omega}_{i}=\mathbf{T}_{i j} \cdot \boldsymbol{\omega}_{i}$,

where $(i, j)=(1,2),(2,1)$. The left hand side of the equations in (20) can be expressed as full derivatives

$\frac{d}{d t}\left(\frac{m_{i} v_{i}^{2}}{2}\right)=\mathbf{F}_{i j} \cdot \mathbf{v}_{i}, \quad \frac{d}{d t}\left(\frac{I_{i} \omega_{i}^{2}}{2}\right)=\mathbf{T}_{i j} \cdot \boldsymbol{\omega}_{i}$

Summing the two equations in (21),

$\sum_{i=1}^{2} \frac{d}{d t}\left(\frac{m_{i} v_{i}^{2}}{2}+\frac{I_{i} \omega_{i}^{2}}{2}\right)=\sum_{(i, j)}\left(\mathbf{F}_{i j} \cdot \mathbf{v}_{i}+\mathbf{T}_{i j} \cdot \boldsymbol{\omega}_{i}\right)$,

where the sum runs over two pairs of values $(i, j)=$ $(1,2),(2,1)$. The sum can be written out as

$$
\begin{aligned}
\frac{d}{d t} & \sum_{i=1}^{2}\left(\frac{m_{i} v_{i}^{2}}{2}+\frac{I_{i} \omega_{i}^{2}}{2}\right) \\
& =\left(\mathbf{F}_{12} \cdot \mathbf{v}_{1}+\mathbf{F}_{21} \cdot \mathbf{v}_{\mathbf{2}}+\mathbf{T}_{12} \cdot \boldsymbol{\omega}_{1}+\mathbf{T}_{21} \cdot \boldsymbol{\omega}_{2}\right) .
\end{aligned}
$$

Recalling Eqs. (1) and (2), Eq. (23) yields:

$\frac{d}{d t} \sum_{i=1}^{2}\left(\frac{m_{i} v_{i}^{2}}{2}+\frac{I_{i} \omega_{i}^{2}}{2}\right)=\mathbf{F}^{T} \mathbf{V}$

We see that the left hand side is the kinetic energy of the two grains. The right hand side is thus the power of the contact forces.

\subsection{Symmetry of the stiffness matrix}

To use the power to find a relation between $\mathbf{C}$ and $\mathbf{C}^{*}$, we need to consider the displacement instead of the velocities. We define

$\mathbf{D}=\int_{t_{0}}^{t} \mathbf{V} d t^{\prime}, \quad \boldsymbol{\delta}=\int_{t_{0}}^{t} \mathbf{v} d t^{\prime}$,

where $t_{0}$ is some reference time. Integrating Eq. (3) leads to

$\delta=\mathbf{C D}$
Next let us suppose that the contact is in a state where the contact forces can be linearized, as described in Eq. (5), leading to

$\mathbf{f}=\mathbf{f}_{0}+\mathbf{k C D}$.

This equation applies both to linear contact laws and to many nonlinear contact laws for infinitesimal displacements. Certain contact laws cannot be linearized; these cases will be discussed below.

Combining with Eq. (7) gives

$\mathbf{F}=\mathbf{F}_{0}+\mathbf{K D}$

with

$\mathbf{K}=\mathbf{C}^{*} \mathbf{k} \mathbf{C}$

We will now show that $\mathbf{K}$ must be symmetric.

Let us consider a cyclic motion of the two spheres.

Let $\mathbf{D}_{1}$ and $\mathbf{D}_{2}$ give two different displacements. Let us consider executing the following cycle, starting from time $t_{0}$ with $\mathbf{D}=0$ :

1. We move slowly to $\mathbf{D}=\mathbf{D}_{1}$.

2. We then move to $\mathbf{D}=\mathbf{D}_{1}+\mathbf{D}_{2}$.

3. We the move to $\mathbf{D}=\mathbf{D}_{2}$.

4. Finally, we move back to $\mathbf{D}=0$.

We will calculate the total work done during one cycle.

In this first step of the cycle:

$W_{1}=\int_{t_{0}}^{t_{1}} P d t=\int_{t_{0}}^{t_{1}} \mathbf{F}^{T} \mathbf{V} d t$

Note that that this integral can be transformed into a line integral in $\mathbb{R}^{12}$ because $\mathbf{D}(t)=\int_{t}^{t_{1}} \mathbf{V} d t$, leading to $d \mathbf{D}=\mathbf{V} d t$ where $d \mathbf{D}$ signifies a vector of infinitesimal components. Using this notation and Eq. (28) yields

$$
\begin{aligned}
W_{1} & =\int_{0}^{\mathbf{D}_{1}}\left[\mathbf{F}_{0}+\mathbf{K D}(t)\right]^{T} d \mathbf{D} \\
& =\mathbf{F}_{0}^{T} \mathbf{D}_{1}+\frac{1}{2} \mathbf{D}_{1}^{T} \mathbf{K D}_{1} .
\end{aligned}
$$

Alternatively, this integral can be done over time. The force law Eq. (28) is independent of velocity, so that we can choose $\mathbf{V}=\mathbf{V}_{1}=\mathbf{D}_{1} / t_{1}$. (after Taking $t_{0}=0$ to lighten the notation). In this notation, $\mathbf{D}(t)=t \mathbf{V}_{1}$, and

$$
\begin{aligned}
W_{1} & =\int_{0}^{t_{1}}\left[\mathbf{F}_{0}+t \mathbf{K} \mathbf{V}_{1}\right]^{T} \mathbf{V}_{1} d t \\
& =t_{1} \mathbf{F}_{0}^{T} \mathbf{V}_{1}+\frac{1}{2} t^{2} \mathbf{V}_{1}^{T} \mathbf{K} \mathbf{V}_{1} \\
& =\mathbf{F}_{0}^{T} \mathbf{D}_{1}+\frac{1}{2} \mathbf{D}_{1}^{T} \mathbf{K D}_{1} .
\end{aligned}
$$


For the remaining steps of the cycle, we will compute integrals, but it is possible to rewrite them as integrals over time. In the second step of the cycle:

$$
\begin{aligned}
W_{2} & =\int_{t_{1}}^{t_{2}} \mathbf{F}^{T} \mathbf{V} d t=\int_{0}^{\mathbf{D}_{2}}\left(\mathbf{D}+\mathbf{D}_{1}\right)^{T} \mathbf{K} d \mathbf{D} \\
& =\mathbf{D}_{2}^{T} \mathbf{K D}_{1}+\frac{1}{2} \mathbf{D}_{2}^{T} \mathbf{K} \mathbf{D}_{2}+\mathbf{F}_{0}^{T} \mathbf{D}_{2} .
\end{aligned}
$$

In the third step of the cycle

$$
\begin{aligned}
W_{3} & =\int_{t_{2}}^{t_{3}} \mathbf{F}^{T} \mathbf{V} d t=\int_{0}^{-\mathbf{D}_{1}}\left(\mathbf{D}+\mathbf{D}_{1}+\mathbf{D}_{2}\right)^{T} \mathbf{K} d \mathbf{D} \\
& =-\mathbf{D}_{1}^{T} \mathbf{K} \mathbf{D}_{2}-\frac{1}{2} \mathbf{D}_{1}^{T} \mathbf{K} \mathbf{D}_{1}-\mathbf{F}_{0}^{T} \mathbf{D}_{1}
\end{aligned}
$$

And finally, the last step of the cycle,

$$
W_{4}=\int_{0}^{-\mathbf{D}_{2}}\left(\mathbf{D}_{2}+\mathbf{D}\right)^{T} \mathbf{K} d \mathbf{D}=-\frac{1}{2} \mathbf{D}_{2}^{T} \mathbf{K D}_{2}-\mathbf{F}_{0}^{T} \mathbf{D}_{2}
$$

Adding the results of all four steps together,

$$
W=\mathbf{D}_{2}^{T} \mathbf{K D}_{1}-\mathbf{D}_{1}^{T} \mathbf{K D}_{2}=\mathbf{D}_{2}\left[\mathbf{K}-\mathbf{K}^{T}\right] \mathbf{D}_{1} .
$$

The total work done vanishes for every choice of $\mathbf{D}_{1}$, $\mathbf{D}_{2}$ if and only if the matrix $\mathbf{K}$ is symmetric. The total work must vanish, otherwise it is possible to generate as much energy as we please by repeating the cycle over and over again, in the correct sense. (If $W<0$ in Eq. (36), we can just run the cycle in reverse to change the sign of $W$ ). If $\mathbf{K}$ is not symmetric, each contact is a possible perpetual motion machine.

Note that contact laws usually include a dissipative part, usually a dashpot, that may compensate for an energy-generating perpetual motion machine, but this solution is difficult to control.

The simplest way to assure that $\mathbf{K}$ is symmetric is to choose a symmetric $\mathbf{k}$ with $\mathbf{C}^{T}=\mathbf{C}^{*}$. This last relation links the contact velocities to the contact forces, and can be used to eliminate certain proposed rolling velocities.

It is possible (but difficult) to construct a symmetric $\mathbf{K}$ with $\mathbf{C}^{T} \neq \mathbf{C}^{*}$. But choosing $\mathbf{C}^{T}=\mathbf{C}^{*}$ has another benefit: it assures that the grain and contact velocities and forces are energetically consistent:

$\mathbf{F}^{T} \mathbf{V}=\left(\mathbf{C}^{*} \mathbf{f}\right)^{T} \mathbf{V}=\mathbf{f}^{T}\left(\mathbf{C}^{* T} \mathbf{V}\right)=\mathbf{f}^{T}(\mathbf{C V})=\mathbf{f}^{T} \mathbf{v}$.

Strictly speaking, this condition is not necessary. Only the forces applied to each grain $\mathbf{F}$ and the grain velocities $\mathbf{V}$ are integrated to obtain grain motion. As we will see in the next section, the quantities $\mathbf{f}$ and $\mathbf{v}$ model physics that we suppose are significant at the contact; that strain is accumulating near the contact point, for example. If $\mathbf{F}^{T} \mathbf{V} \neq \mathbf{f}^{T} \mathbf{v}$, the contact physics used to explain the force law is inconsistent with the actual forces applied to the particles. The contact physics fails to explain the storage or dissipation of energy at the contact.

As mentioned above, certain contact laws cannot be linearized. One example is where a contact stiffness depends on the direction of motion [13]. Another case is the sliding contact: a tangential motion in one direction results in a constant tangential force (equivalent to zero stiffness), while motion in the other direction is associated with non-zero stiffness. These are examples of incremental nonlinearity: the nonlinearity cannot be removed no matter how small the displacements are made. In such cases, the calculation above does not apply, and $\mathbf{K}$ may be asymmetric. Even in these cases, however, it is desirable to have $\mathbf{C}^{T}=\mathbf{C}^{*}$ for the reason described in the preceding paragraph: this choice assures that the incremental nonlinearity is explicitly put into the contact physics, and not some hidden in the way relative velocities are calculated.

\section{Contact surface analysis}

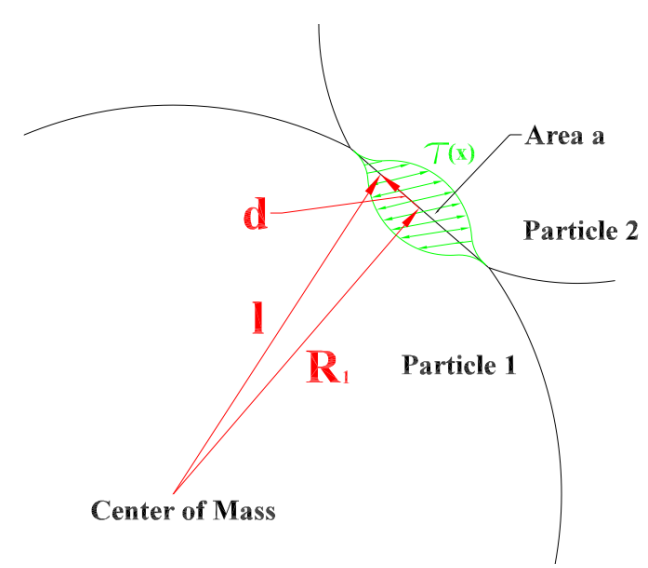

Fig. 2 Interaction between two particles.

In this section, we propose a physically motivated contact model. based on the idea that the two spheres touch at a surface, as shown in Fig. 2. Here, $\mathbf{R}_{i}$ is a vector from the center of particle $i$ to the center of the region of contact. The vector $\mathbf{d}$ specifies the position of a point within the area of contact. 


\subsection{Contact quantities}

As we showed above, a six-dimensional vector suffices to describe the relative velocities. We propose the form

$\mathbf{v}=\left[\begin{array}{c}\mathbf{v}_{c} \\ \boldsymbol{\omega}_{c}\end{array}\right]$

where the three-dimensional vectors $\mathbf{v}_{\mathbf{c}}$ and $\boldsymbol{\omega}_{\mathbf{c}}$ will be called contact velocity and contact angular velocity.

A similar situation holds for the forces: a six-dimensiona vector suffices to specify the contact forces:

$\mathbf{f}=\left[\begin{array}{c}\mathbf{f}_{c} \\ \mathbf{m}_{c}\end{array}\right]$

Here, $\mathbf{f}$ has two components that will be called contact force $\mathbf{f}_{c}$ and contact moment $\mathbf{m}_{c}$. As we showed above, this vector is sufficient to characterize interaction, even though the forces applied to the particles, F, have 12 components.

\subsection{Contact force and torque analysis}

Let us assume that the interaction between two particles is given by a traction, or stress vector, $\boldsymbol{\tau}(\mathbf{x})$ (refer to Fig. 2) on the contact surface created by the deformation of the two spheres at contact. The traction at each point corresponds to the stress tensor times the unit normal vector at this point. Applying Newton's third law, this traction acts on the spheres as

$\tau_{12}(\mathbf{x})=-\tau_{21}(\mathbf{x})=\boldsymbol{\tau}(\mathbf{x})$

where the vector $\boldsymbol{\tau}_{i j}(\mathbf{x})$ is the traction on particle $i$ due to particle $j$. The contact forces acting on each particle become

$\mathbf{F}_{12}=-\mathbf{F}_{21}=\mathbf{f}_{c}=\int_{a} \boldsymbol{\tau}(\mathbf{x}) d a$

Note that that the requirement in Eq. (15) is satisfied for all choices of $\mathbf{f}_{c}$.

The torque produced by the traction of the contact surface $S$ on the particles is given by

$\mathbf{T}_{12}=\int_{a} \ell_{12} \times \boldsymbol{\tau}_{12} d a \quad \mathbf{T}_{21}=\int_{a} \boldsymbol{\ell}_{21} \times \boldsymbol{\tau}_{21} d a$.

where $\boldsymbol{\ell}_{i j}$ is the branch vector connecting the center of mass of the particle $i$ with the point in the contact surface with particle $j$ where the traction is acting. If we replace Eq. (40) into the above equations we get

$\mathbf{T}_{12}=\int_{a} \ell_{12} \times \boldsymbol{\tau} d a \quad \mathbf{T}_{21}=-\int_{a} \boldsymbol{\ell}_{21} \times \boldsymbol{\tau} d a$.
The branch vectors can be written as

$\ell_{12}=\mathbf{R}_{1}+\mathbf{d} \quad \ell_{21}=\mathbf{R}_{2}+\mathbf{d}$

where $\mathbf{d}$ is the vector connecting the center of the contact to the point of application of the traction. Replacing them in the equation above we obtain

$\mathbf{T}_{12}=\mathbf{R}_{1} \times \mathbf{f}_{c}+\mathbf{m}_{c} \quad \mathbf{T}_{21}=-\mathbf{R}_{2} \times \mathbf{f}_{c}-\mathbf{m}_{c}$

where the contact moment is given by

$\mathbf{m}_{c}=\int_{a} \mathbf{d} \times \boldsymbol{\tau} d a$

Note that the expression for the torques in Eq. (45) satisfies Eq. (19) for all choices of $\mathbf{f}_{c}$ and $\mathbf{m}_{c}$.

Finally, let us construct the matrix $\mathbf{C}^{*}$ in Eq. (7). $\mathbf{R}_{i}$ and $\mathbf{f}_{c}$ in Eqs. (45) are defined as:

$\mathbf{R}_{1}=\left[\begin{array}{l}R_{1 x} \\ R_{1 y} \\ R_{1 z}\end{array}\right] \quad \mathbf{R}_{2}=\left[\begin{array}{l}R_{2 x} \\ R_{2 y} \\ R_{2 z}\end{array}\right] \quad \mathbf{f}_{c}=\left[\begin{array}{l}f_{c x} \\ f_{c y} \\ f_{c z}\end{array}\right]$

The cross product $\mathbf{R}_{i} \times \mathbf{f}_{c}$ is defined as $\mathbf{R}_{i}^{*} \mathbf{f}_{c}$.

$\mathbf{R}_{i} \times \mathbf{f}_{c}=\mathbf{R}_{i}^{*} \mathbf{f}_{c}=\left[\begin{array}{ccc}0 & -R_{i z} & R_{i y} \\ R_{i z} & 0 & -R_{i x} \\ -R_{i y} & R_{i x} & 0\end{array}\right]\left[\begin{array}{l}f_{c x} \\ f_{c y} \\ f_{c z}\end{array}\right]$

We rewrite Eq. (45),

$\mathbf{T}_{12}=\mathbf{R}_{1}^{*} \mathbf{f}_{c}+\mathbf{m}_{c} \quad \mathbf{T}_{21}=-\mathbf{R}_{2}^{*} \mathbf{f}_{c}-\mathbf{m}_{c}$

Now we write Eqs. (41) and (45) in a matrix form

$\left[\begin{array}{c}\mathbf{F}_{12} \\ \mathbf{F}_{21} \\ \mathbf{T}_{12} \\ \mathbf{T}_{21}\end{array}\right]=\left[\begin{array}{cc}\mathbf{I} & \mathbf{0} \\ -\mathbf{I} & \mathbf{0} \\ \mathbf{R}_{\mathbf{1}}^{*} & \mathbf{I} \\ -\mathbf{R}_{\mathbf{2}}^{*} & -\mathbf{I}\end{array}\right]\left[\begin{array}{c}\mathbf{f}_{c} \\ \mathbf{m}_{c}\end{array}\right], \quad \mathbf{I}=\left[\begin{array}{lll}1 & 0 & 0 \\ 0 & 1 & 0 \\ 0 & 0 & 1\end{array}\right]$

From this equation, we identify the matrix $\mathbf{C}^{*}$ :

$\mathbf{C}^{*}=\left[\begin{array}{cc}\mathbf{I} & \mathbf{0} \\ -\mathbf{I} & \mathbf{0} \\ \mathbf{R}_{1}^{*} & \mathbf{I} \\ -\mathbf{R}_{2}^{*} & -\mathbf{I}\end{array}\right]$ 


\subsection{Kinematic analysis}

Since the grains are rigid spheres, the velocity of any material point $\mathbf{x}$ of grain $i$ is given by

$\mathbf{v}(\mathbf{x})=\mathbf{v}_{i}+\boldsymbol{\omega}_{i} \times\left(\mathbf{x}-\mathbf{x}_{i}\right)$.

At the surface where the particles touch, there is a velocity jump given by

$$
\begin{aligned}
\Delta \mathbf{v}(\mathbf{x}) & =\mathbf{v}_{1}(\mathbf{x})-\mathbf{v}_{2}(\mathbf{x}) \\
& =\mathbf{v}_{1}-\mathbf{v}_{2}+\boldsymbol{\omega}_{1} \times \boldsymbol{\ell}_{12}-\boldsymbol{\omega}_{2} \times \boldsymbol{\ell}_{21}
\end{aligned}
$$

Expressing $\ell_{12}$ and $\ell_{21}$ as in Eq. (44), we have

$\Delta \mathbf{v}=\mathbf{v}_{1}-\mathbf{v}_{2}+\mathbf{R}_{1} \times \boldsymbol{\omega}_{1}-\mathbf{R}_{2} \times \boldsymbol{\omega}_{2}+\left(\boldsymbol{\omega}_{1}-\boldsymbol{\omega}_{2}\right) \times \mathbf{d}$.

Defining the normal vector at the contact as

$\mathbf{n}=\frac{\mathbf{x}_{1}-\mathbf{x}_{2}}{R_{1}+R_{2}}$,

we have $\mathbf{R}_{1}=R_{1} \mathbf{n}, \mathbf{R}_{2}=-R_{2} \mathbf{n}$, and thus

$\Delta \mathbf{v}=\mathbf{v}_{1}-\mathbf{v}_{2}+\mathbf{n} \times\left(R_{1} \boldsymbol{\omega}_{1}+R_{2} \boldsymbol{\omega}_{2}\right)+\left(\boldsymbol{\omega}_{1}-\boldsymbol{\omega}_{2}\right) \times \mathbf{d}$.

Recall that the contact velocity $\mathbf{v}$ in Eq. (38) is composed of $\mathbf{v}_{c}$ and $\omega_{c}$. Defining

$\mathbf{v}_{c}=\mathbf{v}_{1}-\mathbf{v}_{2}+\mathbf{n} \times\left(R_{1} \boldsymbol{\omega}_{1}+R_{2} \boldsymbol{\omega}_{2}\right)$,

$\boldsymbol{\omega}_{c}=\boldsymbol{\omega}_{1}-\boldsymbol{\omega}_{2}$,

we have

$\Delta \mathbf{v}=\mathbf{v}_{c}+\omega_{c} \times \mathbf{d}$.

This expression will be very useful for the calculation of the power. Before doing this, however, let us obtain the matrix $\mathbf{C}$. Recalling that $\mathbf{v}=\mathbf{C V}$ leads to

$\mathbf{C}=\left[\begin{array}{cccc}\mathbf{I} & -\mathbf{I} & \mathbf{R}_{1}^{*} & -\mathbf{R}_{2}^{*} \\ \mathbf{0} & \mathbf{0} & \mathbf{I} & -\mathbf{I}\end{array}\right]$

Note that $\mathbf{C}^{*}=\mathbf{C}^{T}$, as it should.

Note that we could have done this calculation in reverse. Once $\mathbf{C}$ has been fixed in Eq. (51), then Eq. (59) follows immediately by $\mathbf{C}^{*}=\mathbf{C}^{T}$. Once $\mathbf{C}$ is known, $\mathbf{v}$ is easily obtained from $\mathbf{v}=\mathbf{C V}$. The derivation above to show the physical plausibility of our choice.

Note also that calculating the work done at the contact directly also gives a consistent result:

$$
\begin{aligned}
P & =\int_{a} \boldsymbol{\tau} \cdot \Delta \mathbf{v} d a \\
& =\int_{a} \boldsymbol{\tau} \cdot\left(\mathbf{v}_{c}+\mathbf{d} \times \boldsymbol{\omega}_{c}\right) d a \\
& =\mathbf{f}_{c} \cdot \mathbf{v}_{c}+\int_{a} \boldsymbol{\tau} \cdot\left(\mathbf{d} \times \boldsymbol{\omega}_{c}\right) d a \\
& =\mathbf{f}_{c} \cdot \mathbf{v}_{c}+\int_{a} \boldsymbol{\omega}_{c} \cdot(\tau \times \mathbf{d}) d a \\
& =\mathbf{f}_{c} \cdot \mathbf{v}_{c}+\boldsymbol{\omega}_{c} \cdot \mathbf{m}_{c} \\
& =\mathbf{f}^{T} \mathbf{v}
\end{aligned}
$$

\subsection{The different contact velocities}

Note that all the usual contact velocities are contained in $\mathbf{v}$. The normal velocity is the normal component of $\mathbf{v}_{c}$, and the tangential velocity is the tangential component of $\mathbf{v}_{c}$. The torsion velocity is the normal component of $\boldsymbol{\omega}_{c}$, and the rolling velocity is its tangential components.

If we assume that the modes are independent, the matrix $\mathbf{k}$ can be written as:

$\mathbf{k}=\left[\begin{array}{cccc}k_{n} & 0 & 0 & 0 \\ 0 & k_{t} \mathbf{I}_{2} & 0 & 0 \\ 0 & 0 & \kappa_{n} & 0 \\ 0 & 0 & 0 & \kappa_{t} \mathbf{I}_{2}\end{array}\right], \quad \mathbf{I}_{2}=\left[\begin{array}{ll}1 & 0 \\ 0 & 1\end{array}\right]$

Where $k_{n}, k_{t}, \kappa_{n}$, and $\kappa_{t}$ are the stiffnesses associated to normal, tangential, torsion an rolling motion. More general constitutive relations can be derived by relaxing the assumption that the internal degrees of freedom need to be decoupled.

\section{Consequences}

The two main consequences of our formulation of the generalized interaction between soft spheres are: (i) it rules out some definitions of rolling velocity existing in the literature, and (ii) it restricts the contact model by the condition of the symmetry of the stiffness matrix. We comment briefly on each consequence below.

\subsection{Rolling velocity}

Let us first note that there are different definition of contact rolling in the literature. In particular Bagi and Kuhn [28; 29] surveyed different models of rolling velocity. One proposed definition is given by

$$
\begin{aligned}
\mathbf{v}_{c} & =\mathbf{v}_{1}-\mathbf{v}_{2}+\boldsymbol{\omega}_{1} \times \mathbf{R}_{1}-\boldsymbol{\omega}_{2} \times \mathbf{R}_{2}, \\
\boldsymbol{\omega}_{c}^{B} & =\boldsymbol{\omega}_{1} R_{1}-\boldsymbol{\omega}_{2} R_{2}+\frac{R_{2}-R_{1}}{R_{1}+R_{2}}\left(\mathbf{v}_{1}-\mathbf{v}_{2}\right) .
\end{aligned}
$$

The contact velocity is unchanged, but the angular contact velocity mixes angular and translational velocities. The relations are based on an early derivation of Iwashita-Oda [8] and it is proven that they are objective. The $\mathbf{C}$ matrix associated to Eq. (62) is given by

$$
\mathbf{C}=\left[\begin{array}{cccc}
\mathbf{I} & -\mathbf{I} & \mathbf{R}_{1}^{*} & -\mathbf{R}_{2}^{*} \\
R \mathbf{I}-R \mathbf{I} & R_{1} \mathbf{I} & -R_{2} \mathbf{I}
\end{array}\right] \quad R=\frac{R_{2}-R_{1}}{R_{1}+R_{2}}
$$

If this rolling velocity is used to generate forces that oppose the rolling motion, then torques and forces must also be mixed. But in [29], this rotational velocity is used only to analyze grain motion, so our work does 
not directly impact that paper. However, we hope the authors will not give into the temptation to use this rolling velocity to generate torques!

\subsection{Coupling contact velocities}

Our work also has consequences for models that couple the different degrees of freedom. The symmetry of $\mathbf{K}$ is assured if $\mathbf{C}=\mathbf{C}^{* T}$ and if $\mathbf{k}$ is symmetric. This symmetry condition reduces the number of component of the stiffness matrix to 21. Non-diagonal elements may indicate that the different deformation modes are coupled. For example, rolling deformation can affect normal force. But if this is so, then the symmetry of $\mathbf{k}$ requires that the normal force affect the rolling resistance.

\section{Summary and conclusions}

Contact laws have been a major concern since the early days of particle simulations and e.g. for non-spherical particles. Major contributions were based on the innovation triggered in the group around Hans Herrmann, where the authors also learned a lot.

In summary, we presented a general formulation of interaction between soft spheres based on objectivity and conservation principles. We first used Newtons third law on tractions to obtain the relation between forces and torques with contact forces and contact moment. Examining the power generated at contacts leads to another, hitherto unrecognized, constraint on the interaction: the stiffness matrix must be symmetric, unless their is incremental nonlinearity. We then present a simple contact model for two spheres that includes rolling and torsion that illustrates these constraints.

From our derivation we conclude that some expressions of rolling velocity existing in the literature should be ruled out when calculating rolling resistance, to avoid numerical problems of energy conservation. Our work also shows how different degrees of freedom at the contact could be couples, while still respecting the basic mechanical laws. Coupled contact laws can be useful to mimic the effect of particle shape via rolling resistance [16], to model the complex interaction of particles in cohesive power [13], or in meso-scale simulations where a discrete element represent an ensemble of particles $[30 ; 31]$

Acknowledgements The authors thank Stefan Luding for major contributions to this paper and his patient encouragement. We acknowledge scientific discussion with Hans Herrmann, Lothar Brendel, Hans Muhlhaus, Antoinette Tordesillas, Yucang Wang, and Thomas Weinhart on rolling resistance. We thank Shumiao Chen and Coraline Chiew for editing earlier versions of this manuscript. We thank Falk Wittel for applying the last kick needed to get this paper finished. We express special dedication to Hans Herrmann for his enthusiastic approach to science and the time we spent in one of his many institutes. The project was funded by the USYD Civil Engineering Research Development Scheme, 2013.

\section{References}

1. P. A. Cundall and O. D. Strack, Geotechnique 29, 47 (1979).

2. H. J. Herrmann, J. Hovi, and S. Luding, NATO-ASI Series E 350, Kluwer academic publishers, Dordrecht (1998).

3. H. M. Jaeger, S. R. Nagel, and R. P. Behringer, Reviews of Modern Physics 68, 1259 (1996).

4. C. Goldenberg and I. Goldhirsch, Physical Review E 77, 041303 (2008).

5. D. M. Mueth, H. M. Jaeger, and S. R. Nagel, Physical Review E 57, 3164 (1998).

6. Y. Fukumoto, H. Sakaguchi, and A. Murakami, Granular Matter 15, 175 (2013).

7. M. Oda, J. Konishi, and S. Nemat-Nasser, Mechanics of materials 1, 269 (1982).

8. K. Iwashita and M. Oda, Journal of engineering mechanics 124, 285 (1998)

9. R. Fuchs, T. Weinhart, J. Meyer, T. Staedler, X. Jiang, and S. Luding, arXiv preprint arXiv:1401.2600 (2014).

10. K. Iwashita and M. Oda, Powder Technology 109, 192 (2000).

11. A. Tordesillas and D. Walsh, Powder Technology 124, 106 (2002).

12. R. Kirsch, U. Bröckel, L. Brendel, and J. Török, Granular Matter 13, 517 (2011).

13. S. Luding, Granular Matter 10, 235 (2008).

14. Y. Wang and P. Mora, Pure and Applied Geophysics 165, 609 (2008).

15. K. Iwashita and M. Oda, Journal of engineering mechanics 124, 286 (1998).

16. A. Tordesillas and D. Walsh, Powder Technology 124, 106 (2002).

17. H. M. Shodja and E. G. Nezami, Int. J. Numer. Anal. Meth. Geomech. 27, 403 (2003).

18. K. Bagi and M. R. Kuhn, J. Appl. Mech 71, 493 (2004).

19. M. R. Kuhn and K.Bagi, J. Eng. Mech. 130, 826 (2004).

20. F. Alonso-Marroquin, I. Vardoulakis, H. Herrmann, D. Weatherley, and P. Mora, Physical Review E 74, 031306 (2006).

21. J. Ai, J.-F. Chen, J. M. Rotter, and J. Y. Ooi, Powder Technology 206, 269 (2011).

22. A. Mohamed and M. Gutierrez, Granular Matter 12, 527 (2010).

23. L. Brendel, J. Török, R. Kirsch, and U. Bröckel, Granular Matter 13, 777 (2011).

24. M. Jiang, H.-S. Yu, and D. Harris, Computers and Geotechnics 32, 340 (2005).

25. Y. C. Wang, F. Alonso-Marroquin, S. Xue, and J. Xie, Particuology Accepted, (2014).

26. J. Roux, Physical Review E 61, 6802 (2000).

27. T. R. Chandrupatla, A. D. Belegundu, T. Ramesh, and C. Ray, Introduction to finite elements in engineering (PrenticeHall Englewood Cliffs, New Jersey, 1991).

28. K. Bagi and M. R. Kuhn, Journal of applied mechanics 71, 493 (2004).

29. M. R. Kuhn and K. Bagi, International journal of solids and structures 41, 5793 (2004). 
30. A. Singh, V. Magnanimo, and S. Luding, Powder Technology (2013).

31. N. J. Brown, J.-F. Chen, and J. Y. Ooi, Granular Matter 1 (2014). 